\title{
Periodicity in some light curves of the solar analogue V352 Canis Majoris * (Research Note)
}

\author{
P. Kajatkari ${ }^{1}$, L. Jetsu ${ }^{1}$, E. Cole ${ }^{1}$, T. Hackman ${ }^{1,2}$, G. W. Henry ${ }^{3}$, S.-L. Joutsiniemi ${ }^{1}$, J. Lehtinen ${ }^{1,4}$, V. Mäkelä ${ }^{1}$, \\ S. Porceddu ${ }^{1}$, K. Ryynänen ${ }^{1}$, and V. Solea ${ }^{1}$ \\ ${ }^{1}$ Department of Physics, Gustaf Hällströmin katu 2a (PO Box 64), University of Helsinki, 00014 Helsinki, Finland \\ e-mail: lauri.jetsu@helsinki.fi \\ 2 Finnish Centre for Astronomy with ESO, University of Turku, Väisäläntie 20, 21500 Piikkiö, Finland \\ 3 Center of Excellence in Information Systems, Tennessee State University, 3500 John A. Merritt Blvd., Box 9501, Nashville, \\ TN 37209, USA \\ ${ }^{4}$ Nordic Optical Telescope, 38700 Santa Cruz de la Palma, Spain
}

Received 14 October 2013 / Accepted 24 March 2015

\section{ABSTRACT}

\begin{abstract}
Aims. We applied the Continuous Period Search (CPS) method to $14 \mathrm{yr}$ of $V$-band photometry of the active G6.5 solar analog V352 CMa. Our aim was to show that CPS can successfully model the presence or absence of periodicity in low-amplitude light curves.

Methods. CPS computes values for the mean brightness, photometric period, amplitude and minimum of selected datasets. We also applied the Power Spectrum Method (PSM) to these datasets and compared the performance of this frequently applied method to that of CPS.

Results. We found an apparent $11.7 \pm 0.5 \mathrm{yr}$ cycle in the mean brightness. The mean of the individual photometric rotation periods is $7.24 \pm 0.22$ days. The lower limit for the differential rotation coefficient is $|k|>0.12$, assuming that period changes in V352 CMa follow the solar pattern. The Kuiper method detected stable, active longitudes rotating with a period of $7.157 \pm 0.002$ days, from the epochs of light minimum, but these structures vanished after the year 2009. CPS performed better than the traditional PSM, because the latter assumes a sinusoidal model for the data even when this was not correct.
\end{abstract}

Key words. methods: data analysis - stars: activity - starspots - stars: individual: V352 CMa

\section{Introduction}

V352 CMa (HD 43162) was among the first 384 bright, extremeultraviolet sources detected by the ROSAT satellite (Shara et al. 1993). Its metallicity and space motion indicate that it belongs to the young disk population (Eggen 1995). It was included in a list of 38 nearby, young, single solar analogs having ages between 0.2 and 0.8 Gyr (Gaidos 1998). We collected values for some of its physical parameters from the literature and list them in Table 1. Strong Ca H\&K emission $\left(R_{\mathrm{HK}}^{\prime}\right)$, high X-ray luminosity $\left(R_{\mathrm{X}}\right)$, and rapid rotation $(v \sin i)$ all indicate that HD 43162 is a young star. Its high lithium abundance also supports a young age (Gaidos 1998; Santos et al. 2004a). However, the metallicity $([\mathrm{Fe} / \mathrm{H}])$ estimates are inconsistent with HD 43162's youth (Table 1). Except for Gaidos et al. (2000) and Gaidos \& Gonzalez (2002), the effective surface temperature $\left(T_{\text {eff }}\right)$ and gravity $(\log g)$ estimates agree and support the spectral type G6.5 V (Gray et al. 2006).

* The analysed photometry and numerical results of the analysis are only available at the CDS via anonymous ftp to cdsarc.u-strasbg.fr (130.79.128.5) or via http://cdsarc.u-strasbg.fr/viz-bin/qcat?J/A+A/577/A84
V352 CMa was classified as a member of a stellar kinematical group (hereafter SKG) of 19 stars by Jeffries \& Jewell (1993). It was later identified as a member of the kinematic group IC 2391, a more recently identified SKG containing 29 stars. The estimated age of IC 2391 is 45 Myr (Nakajima et al. 2010; Maldonado et al. 2010; Nakajima \& Morino 2012). However, only two stars in IC 2391 are among the 19 members of the original SKG defined by Jeffries \& Jewell (1993): V352 CMa and LQ Hya. This demonstrates how difficult it is to confirm the membership in any particular SKG.

Christian et al. (2003) observed an extreme ultraviolet flare in an M3.5 V star (EUVE J0613-23.9B) located just 2.5 away from V352 CMa (EUVE J0613-23.9). This M3.5 V star was identified as a binary companion of V352 CMa (Raghavan et al. 2010). About $90 \%$ of the ROSAT source positions are accurate to $1^{\prime}$ or better, while $100 \%$ are within $2 ! 1$ of their catalogued positions (Shara et al. 1993). The distance between V352 CMa and its M3.5 companion is 2.5, close to the ROSAT 2'1 limit. This companion was excluded from our photometric measurements of V352 CMa by the 55" focal-plane diaphragm of the photometer. The orbital plane of the companion must be nearly perpendicular to the line of sight because $v_{\text {rad }}$ appears to be constant (Table 1). If the rotation axis of $\mathrm{V} 352 \mathrm{CMa}$ is perpendicular 
Table 1. Physical parameters of V352 CMa.

\begin{tabular}{|c|c|c|c|c|c|c|c|c|c|c|c|c|c|c|c|}
\hline $\begin{array}{l}T_{\text {eff }} \\
{[\mathrm{K}]} \\
\end{array}$ & Ref. & $\begin{array}{c}\log g \\
{\left[\mathrm{~cm} \mathrm{~s}^{-2}\right]}\end{array}$ & Ref & $\begin{array}{c}v_{\text {rad }} \\
{\left[\mathrm{km} \mathrm{s}^{-1}\right]}\end{array}$ & Ref. & $\begin{array}{c}v \sin i \\
{\left[\mathrm{~km} \mathrm{~s}^{-1}\right]} \\
\end{array}$ & \multicolumn{2}{|c|}{ Ref. ${ }^{[\mathrm{Fe} / \mathrm{H}]}$} & Ref. & $\begin{array}{c}\text { Age } \\
{[\mathrm{Myr}]}\end{array}$ & Ref. & $R_{\mathrm{HK}}^{\prime}$ & \multicolumn{3}{|c|}{$R_{\mathrm{X}}$} \\
\hline 5480 & 7 & 4.57 & 10 & $22.7 \pm 0.7$ & 1 & $6 \pm 2$ & 6 & -0.15 & 4 & $200-800$ & 5 & -4.40 & 7 & -4.26 & 5 \\
\hline 5593 & 8 & 4.10 & 11 & $21.69 \pm 0.16$ & 2 & 5.7 & 7 & -0.16 & 9 & $70-800$ & 7 & -4.40 & 12 & -4.31 & 7 \\
\hline 5630 & 10 & 4.48 & 13 & $22.6 \pm 0.6$ & 3 & 5.49 & 13 & -0.02 & 10 & 575 & 12 & -4.48 & 18 & -4.29 & 21 \\
\hline 5473 & 11 & 4.49 & 18 & $21.3-21.8$ & 6 & 9.63 & 22 & -0.11 & 11 & $<1000$ & 14 & -4.39 & 21 & -4.39 & 22 \\
\hline 5633 & 13 & 4.52 & 23 & 21.7 & 7 & & & -0.01 & 13 & $<6500$ & 19 & & & & \\
\hline 5585 & 15 & & & $21.9 \pm 0.2$ & 16 & & & -0.10 & 15 & $33-35,280$ & 21 & & & & \\
\hline 5571 & 18 & & & $21.91 \pm 0.09$ & 17 & & & -0.10 & 19 & 5286 & 24 & & & & \\
\hline 5584 & 19 & & & $22.23 \pm 0.09$ & 21 & & & -0.04 & 20 & $<45$ & 25 & & & & \\
\hline 5590 & 23 & & & & & & & 0.02 & 23 & & & & & & \\
\hline & & & & & & & & 0.02 & 24 & & & & & & \\
\hline
\end{tabular}

References. (1) Beavers \& Eitter (1981); (2) Andersen et al. (1985); (3) Beavers \& Eitter (1986); (4) Eggen (1995); (5) Gaidos (1998); (6) Cutispoto et al. (1999); (7) Gaidos et al. (2000); (8) Decin et al. (2000); (9) Haywood (2001); (10) Santos et al. (2001); (11) Gaidos \& Gonzalez (2002); (12) Wright et al. (2004); (13) Santos et al. (2004a); (14) Santos et al. (2004b); (15) Nordström et al. (2004); (16) Gontcharov (2006); (17) Abt \& Willmarth (2006); (18) Gray et al. (2006); (19) Holmberg et al. (2009); (20) Árnadóttir et al. (2010); (21) Maldonado et al. (2010); (22) Martínez-Arnáiz et al. (2010); (23) Brugamyer et al. (2011); (24) Fernandes et al. (2011); (25) Nakajima \& Morino (2012).

to this orbital plane, the light curve amplitudes would be supressed by our high-inclination viewing angle. V352 CMa is one of 11 stars with debris disks detected with the Spitzer Space Telescope (Kóspál et al. 2009). However, it is the only star in this sample without a known planet.

Cutispoto et al. (1999) determined the photometric rotation period of V352 CMa, $P_{\text {phot }}=7.2 \pm 0.2$, from a light curve with a low 0.03 peak-to-peak amplitude. Gaidos et al. (2000) found no periodicity in their photometry, which were the same data as our Season 1 of this paper. HIPPARCos photometry also failed to detect any periodicity (Koen \& Eyer 2002, $n=228$ observations). Wright et al. (2004) derived $P_{\text {phot }} \approx 8^{\mathrm{d}}$ from observed $B-V$ and $R_{\mathrm{HK}}^{\prime}$ values. In this paper, we analyse long-term photometry of V352 CMa with Continuous Period Search (CPS) method (Lehtinen et al. 2011). The previous studies have indicated that periodicity may be present or absent in any particular observing season. We show that CPS is an ideal method for analysing this type of challenging data. We also compare CPS to Power Spectrum Method (PSM) formulated by Horne \& Baliunas (1986).

\section{Observations}

The photometry of our target star S = V352 CMa was obtained with the T3 $0.4 \mathrm{~m}$ automated photoelectric telescope (APT) at Fairborn Observatory in Arizona. The observations were made during 14 observing seasons between December 23rd, 1998 $(\mathrm{HJD}=2451$ 170.8) and March 17th, $2012(\mathrm{HJD}=2456003.6)$. The comparison star was $\mathrm{C}=\mathrm{HD} 43879$ (F5V, $V=7$. 51), which is a visual double with $V=7.3$ and $11 \mathrm{~m} .2$ components. The angular separation of the two components is 6.'7, so both fall easily within in the $55^{\prime \prime}$ focal-plane diaphragm of the photometer. The check star was $\mathrm{K}=\mathrm{HD} 43429$ (K1 III, $V=5$ m.99). The standard Johnson differential magnitudes, $\Delta V_{\mathrm{S}-\mathrm{C}}$, are plotted in Fig. 1a. The mean $(m)$ and the standard deviation $(s)$ of these $n=1257$ observations are $m \pm s=-1.0929 \pm 0$. 0096 . The lower panel displays the $\Delta V_{\mathrm{K}-\mathrm{C}}$ differential magnitudes. The standard deviation of these $\Delta V_{\mathrm{K}-\mathrm{C}}$ observations $(n=1025)$ is 0.0079 , which we take as an estimate of the precision of a single observation. We used this estimate to test the constant brightness hypothesis for $\Delta V_{\mathrm{S}-\mathrm{C}}$. The result, $\chi^{2}=1854$, for these $\Delta V_{\mathrm{S}-\mathrm{C}}$ observations $(n=1257)$ indicates that the variability implied by the V352 CMa observations is real. There are also clear trends in

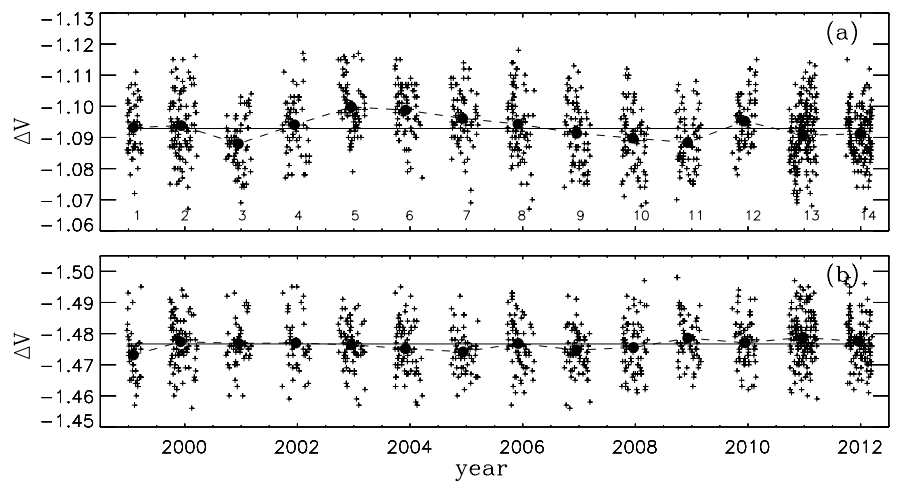

Fig. 1. Differential photometry of V352 CMa. a) All $\Delta V_{\mathrm{S}-\mathrm{C}}$ data (crosses), their mean level (continuous line) and the seasonal $\Delta V_{\mathrm{S}-\mathrm{C}}$ means (filled circles) connected with dashed lines. b) All $\Delta V_{\mathrm{K}-\mathrm{C}}$ data. The magnitude scale and the notations are as in a).

the $n=14$ seasonal $\Delta V_{\mathrm{S}-\mathrm{C}}$ means having $s=0$ m 0036 (Fig. 1a: filled circles). The respective seasonal $\Delta V_{\mathrm{K}-\mathrm{C}}$ means are more stable with $s=0$. 0016 (Fig. 1b: filled circles). More detailed information of the operation of the T3 $0.4 \mathrm{~m}$ APT and the data reduction procedures can be found, e.g., in Henry (1999) and Fekel \& Henry (2005).

\section{CPS method}

CPS was formulated by Lehtinen et al. (2011), where it is described in detail. Here, we only briefly describe CPS, because we have already applied it to the photometry of several stars (Hackman et al. 2011, 2013; Lehtinen et al. 2012; Kajatkari et al. 2014). CPS divides the data into segments (SEG) and datasets (SET). The maximum length for a dataset is $\Delta T_{\max }=30^{\mathrm{d}}$. Each dataset has to contain at least $n \geq n_{\min }=12$ observations $y_{i}=\Delta V_{\mathrm{S}-\mathrm{C}}\left(t_{i}\right)$. This division gives 14 segments (i.e., observing seasons). Our notation for the mean of the $n$ observing times $t_{i}$ of a dataset is $\tau$.

CPS model is a $K$ th order Fourier series

$\hat{y}\left(t_{i}\right)=\hat{y}\left(t_{i}, \bar{\beta}\right)=M+\sum_{k=1}^{K}\left[B_{k} \cos \left(k 2 \pi f t_{i}\right)+C_{k} \sin \left(k 2 \pi f t_{i}\right)\right]$. 
P. Kajatkari et al.: Periodicity in some light curves of the solar analogue V352 Canis Majoris (RN)

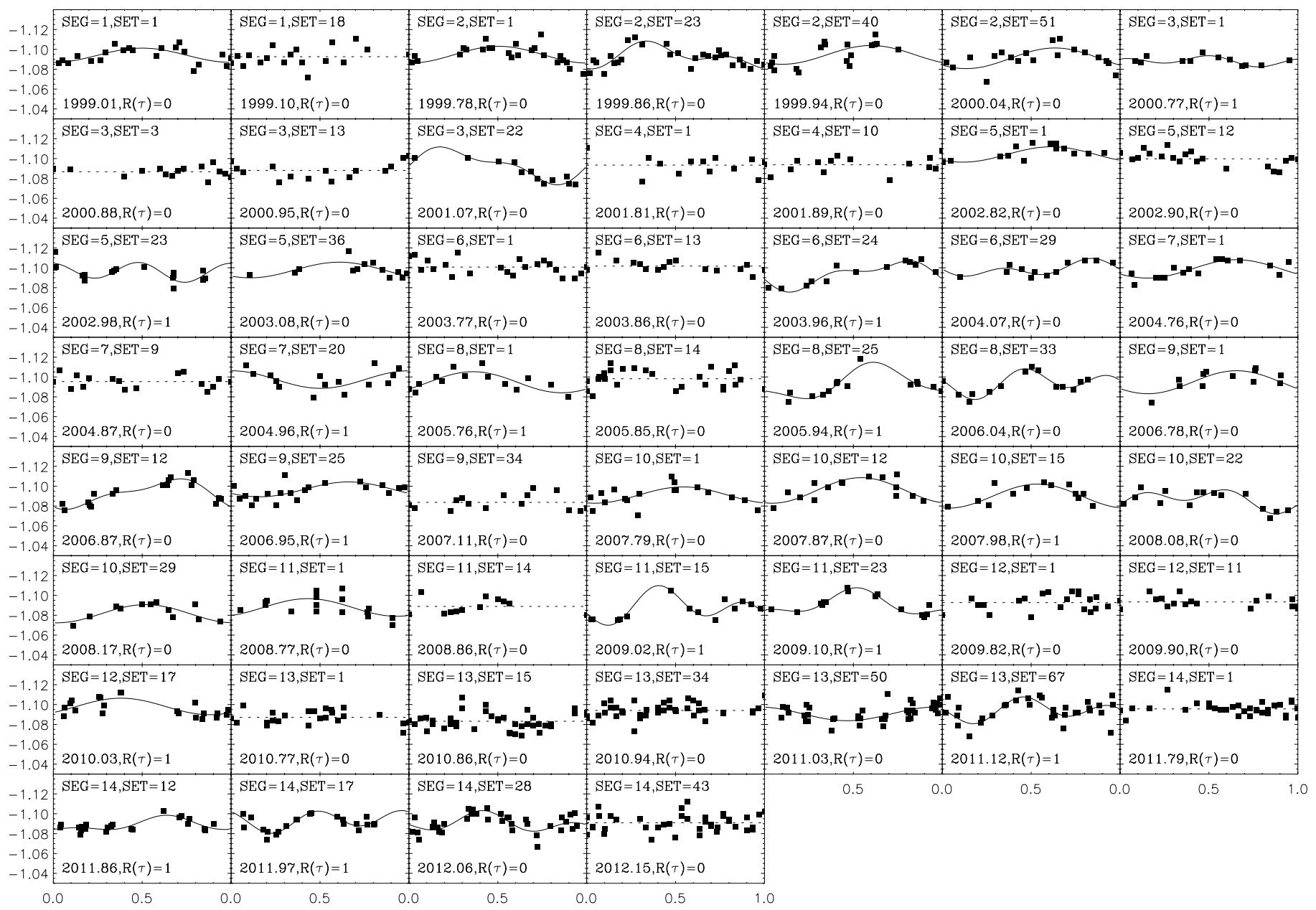

Fig. 2. Light curves of all independent datasets $(\operatorname{IND}(\tau)=1)$. All notations are explained in the 3rd paragraph of Sect. 4.1

Of the free parameters $\bar{\beta}=\left[M, B_{1}, \ldots, B_{K}, C_{1}, \ldots, C_{K}, f\right]$, the physically meaningful ones are the mean, $M(\tau)$ and the period, $P(\tau)=f^{-1}(\tau)$. The other free parameters $B_{1}, \ldots, B_{K}, C_{1}, \ldots, C_{K}$ give the total amplitude of the model, $A(\tau)$, as well as the epochs of the primary and secondary minima in time, $t_{\min , 1}(\tau)$ and $t_{\min , 2}(\tau)$

CPS uses a Bayesian information criterion to choose the best modelling order $K$ for each dataset (Lehtinen et al. 2011, Eq. (6)). We tested orders $0 \leq K \leq 2$.

CPS results correlate for temporally overlapping subsets because they contain common data. To eliminate such correlation, we selected a sequence of independent datasets with no common data. The notations $\operatorname{IND}(\tau)=1$ and 0 are used for independent and not independent (i.e. overlapping) datasets, respectively.

The error estimates for $M(\tau), A(\tau), P(\tau), t_{\min , 1}(\tau)$ and $t_{\min , 2}(\tau)$ were determined with the bootstrap method. This bootstrap was also used to identify the reliable and unreliable light curve parameters. Our notations are $R(\tau)=0$ for reliable estimates and $R(\tau)=1$ for unreliable estimates.

For reliable datasets, CPS also gives the time scale of change of the light curve, $T_{\mathrm{C}}(\tau)$, i.e., the time that the light curve model is valid for the subsequent datasets.

\section{Results}

The CPS results are published electronically at the CDS. We use the same format specified by Lehtinen et al. (2011, Appendix A).

\subsection{General results}

The total number of modelled datasets is 485 . Of these, no periodicity was detected in 178 datasets $(K=0)$, where the best model was constant brightness $\hat{y}\left(t_{i}\right)=M(\tau)$. Periodicity was detected in 307 datasets. The best orders were $K=1$ for 170 datasets and $K=2$ for 137 datasets.

The light curves of all independent datasets are shown in Fig. 2. The continuous lines display the periodic curves $(K \geq 1)$. The phases were first computed from $\phi_{1}=$ $\operatorname{FRAC}\left[\left(t-t_{\min , 1}(\tau)\right) / P(\tau)\right]$, where FRAC $[x]$ removes the integer part of its argument $x$. Then the phases $\phi_{\mathrm{al}, 1}$ of the $t_{\min , 1}(\tau)$ epochs were computed from the constant period ephemeris HJD $2451178.1245+7$ d $158 \mathrm{E}$ (see Sect. 4.4). Finally, the data and the light curves were plotted as a function of $\phi=\phi_{1}+\phi_{\mathrm{al}, 1}$. The dashed lines display the aperiodic curves $(K=0)$, where the "phases" are $\phi_{i}=\left(t_{i}-t_{1}\right) /\left(t_{n}-t_{1}\right)$. The SEG, SET, $\tau$ and $R(\tau)$ values are also given in each panel.

The results for $M(\tau), A(\tau), P(\tau), t_{\min , 1}(\tau)$ and $t_{\min , 2}(\tau)$ are summarised below.

\begin{tabular}{cllll}
\hline \hline & $\operatorname{IND}(\tau)=1$ & $\operatorname{IND}(\tau)=1$ & $\operatorname{IND}(\tau)=0$ & $\operatorname{IND}(\tau)=0$ \\
& $R(\tau)=0$ & $R(\tau)=1$ & $R(\tau)=0$ & $R(\tau)=1$ \\
\hline$M(\tau)$ & $n=39[\mathbf{\square}]$ & $n=14[\square]$ & $n=331[\times]$ & $n=101[\times]$ \\
$A(\tau)$ & $n=20[\mathbf{\square}]$ & $n=14[\square]$ & $n=172[\times]$ & $n=101[\times]$ \\
$P(\tau)$ & $n=20[\mathbf{\square}]$ & $n=14[\square]$ & $n=172[\times]$ & $n=101[\times]$ \\
$t_{\min , 1}(\tau)$ & $n=20[\mathbf{\square}]$ & $n=14[\square]$ & $n=172[\times]$ & $n=101[\times]$ \\
$t_{\min , 2}(\tau)$ & $n=5[\mathbf{\Delta}]$ & $n=8[\triangle]$ & $n=65[\times]$ & $n=47[\times]$ \\
\hline
\end{tabular}




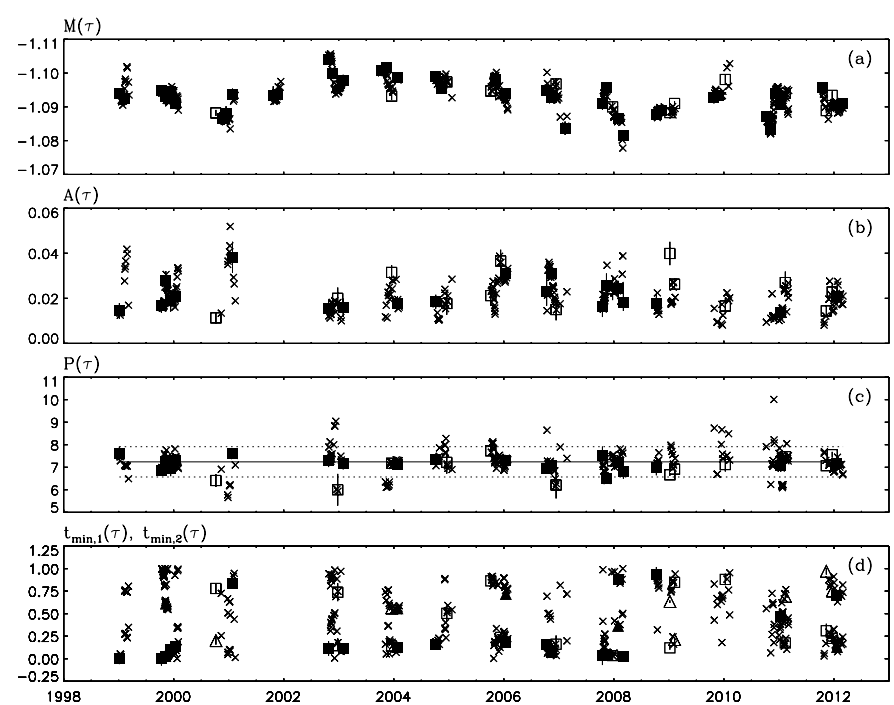

Fig. 3. a) Mean $M(\tau)$, b) amplitude $A(\tau)$, c) period $P(\tau)$ and d) phases of the primary $t_{\min , 1}(\tau)$ and secondary $t_{\min , 2}(\tau)$ minima. The symbols are explained in the 3rd paragraph of Sect. 4.1. The horizontal lines in c) represent the $P_{\mathrm{w}} \pm 3 \Delta P_{\mathrm{w}}$ levels. The phases in d) were calculated from HJD $2451178.1245+7^{\mathrm{d}} .158 \mathrm{E}$.

The symbols given in parenthesis are used in Figs. 3a-d, where error bars are displayed only for $\operatorname{IND}(\tau)=1$ datasets.

The light curve shape of V352 CMa evolved significantly only within 37 datasets, where the $T_{\mathrm{C}}$ mean was 51 days. This mean exceeded 65 days in the other 333 datasets, where the light curve did not change within the segment. These values, $T_{\mathrm{C}}=51$ and 65 days, indicate that the chosen length of the datasets, $\Delta T_{\max }=30^{\mathrm{d}}$, should give reliable CPS results.

\subsection{Activity cycles}

The long-term $M(\tau)$ and $A(\tau)$ changes of V352 CMa are shown in Fig. 3, panels a) and b).

PSM has been applied to search for activity cycles in chromospheric Ca II H\&K emission line data (e.g., Baliunas et al. 1995). Rodonò et al. (2000) applied this method to the following light curve parameters: $M(\tau)$ (axisymmetric part of spot distribution), $A(\tau)$ (non-axisymmetric part of spot distribution), $M(\tau)-A(\tau) / 2$ (minimum spotted area) or $M(\tau)+A(\tau) / 2$ (maximum spotted area). We applied PSM to the same independent and reliable estimates of V352 CMa. The false alarm probability of the best cycle, $P_{\mathrm{C}}=11.7 \pm 0.5 \mathrm{yr}$ for the $n=39$ values of $M(\tau)$, is $F=0.01$. This cycle probability is $1-F=0.99$. The cycles for the $n=20$ values of $A(\tau), M(\tau)-A(\tau) / 2$ and $M(\tau)+A(\tau) / 2$ reached only $F \geq 0.26$.

\subsection{Differential rotation}

The weighted mean of the independent and reliable $P(\tau)(n=$ 20) estimates is $P_{\mathrm{w}} \pm \Delta P_{\mathrm{w}}=7.24 \pm 0.22$. This range of period changes, $P_{\mathrm{w}} \pm 3 \Delta P_{\mathrm{w}}$, (Fig. 3c: dotted lines) gave $Z=$ $6 \Delta P_{\mathrm{w}} / P_{\mathrm{w}}=0.19 \equiv 19 \%$ (Lehtinen et al. 2011, Eq. (14)). The average of the light curve half amplitude $A(\tau) / 2$ in these datasets was only 0 . 011 . The precision of the photometry, $\sigma_{\mathrm{N}}=0.07$, gives an amplitude to noise ratio of $A / N \lesssim 2$. Even if the spurious period changes were $Z_{\text {spu }} \geq 0.15$, the estimated real physical changes could be $Z_{\text {phys }}=\left(Z^{2}-Z_{\text {spu }}^{2}\right)^{-1 / 2} \approx 0.12$ (Lehtinen et al. 2011, Table 3 and Eq. (15)). Here, we assume that the solar law of surface differential rotation can be applied to V352 CMa and that the period $P(\tau)$ changes can be used as reliable tracer surface differential rotation. We used $k \approx Z_{\text {phys }} / h$, where the minimum and maximum latitudes of spot activity were $b_{\min }$ and $b_{\max }$, and $h=\sin ^{2} b_{\max }-\sin ^{2} b_{\min }$ (Jetsu et al. 2000). The maximum value, $h=1$, would be reached if spots formed at all latitudes between the equator and pole of V352 CMa. All other alternatives give $h<1 \Rightarrow|k|>Z_{\text {phys }} \approx 0.12$. This result is comparable to the solar value $k=0.20$, where sunspots typically form at latitudes $-30^{\circ} \leq b \leq 30^{\circ}$ (i.e., $h=0.25$ ).

\subsection{Active longitudes}

We applied the non-weighted Kuiper test (Jetsu \& Pelt 1996) to the reliable primary minima $t_{\min , 1}(\tau)$ of 20 independent datasets. The test range was between $0.85 P_{w}=6.0$ and $1.15 P_{w}=8.1$. The critical level for the best period $P_{\mathrm{al}, 1}=7.158 \pm 0.002$ was $Q=0.004$. The test for the reliable $t_{\min , 1}(\tau)$ and $t_{\min , 2}(\tau)$ ( $n=25$ ) of all independent datasets gave the same result, $P_{\mathrm{al}, 1,2}=7 \mathrm{~d} .158 \pm 0.002$, but the critical level for this period had lower significance, $Q=0.056$. The phases of all primary and secondary minima are shown in Fig. 3d, computed from the ephemeris HJD $2451178.1245+7$ d $158 \mathrm{E}$. The phases of $t_{\min , 1}$ were very stable between 1998 and 2009 (Fig. 3d: filled squares). However, this long-lived structure vanished after 2009 when the light curve amplitudes $A(\tau)$ decreased.

\subsection{Comparison of CPS and PSM}

We also applied PSM to all datasets analysed with CPS. Our aim was to check how often these two methods detected the same periodicity. We use the abbreviation " $C_{0}$ " for the case

$P_{\mathrm{CPS}}-\sigma_{P_{\mathrm{CPS}}} \leq P_{\mathrm{PSM}} \leq P_{\mathrm{CPS}}+\sigma_{P_{\mathrm{CPS}}}$,

where $P_{\mathrm{CPS}} \pm \sigma_{P_{\mathrm{CPS}}}$ and $P_{\mathrm{PSM}}$ were the periods detected with CPS and PSM, respectively. For six different criteria, we checked the number of cases where $C_{0}$ was true

\begin{tabular}{clll}
\hline \hline Criterion & $K=1$ or $2[\%]$ & $K=1[\%]$ & $K=2[\%]$ \\
\hline$A(\tau)>0$ & $173 / 307 \equiv 56$ & $141 / 170 \equiv 83$ & $32 / 137 \equiv 23$ \\
$A(\tau)>0.015$ & $129 / 240 \equiv 54$ & $97 / 112 \equiv 87$ & $32 / 128 \equiv 25$ \\
$A(\tau)>0.030$ & $15 / 40 \equiv 38$ & $3 / 3 \equiv 100$ & $12 / 37 \equiv 32$ \\
\hline$F<0.5$ & $152 / 258 \equiv 59$ & $126 / 149 \equiv 85$ & $26 / 109 \equiv 24$ \\
$F<0.1$ & $52 / 86 \equiv 60$ & $41 / 42 \equiv 98$ & $11 / 44 \equiv 25$ \\
$F<0.05$ & $25 / 36 \equiv 69$ & $18 / 18 \equiv 100$ & $7 / 18 \equiv 39$ \\
\hline
\end{tabular}

Case $C_{0}$ was true only in $56 \%$ cases for all periodicity detections $(n=307: K=1$ or $2, A(\tau)>0.00)$. This occurred more often for $K=1(83 \%)$ than for $K=2(23 \%)$ models. These fractions increased for larger amplitudes, $A(\tau)>0.015$. For the highest amplitudes, $A(\tau)>0.030$, case $C_{0}$ was true for all $K=1$ models (100\%) but not for all $K=2$ models (32\%).

As expected, the probability for case $C_{0}$ being true also increased when the false alarm probability $F$ decreased. For $F<0.05$, case $C_{0}$ was true for all $K=1$ models $(100 \%)$, but again not for all $K=2$ models (39\%).

In conclusion, PSM did not always detect the correct period for the $K=2$ light curves. This was true also for high amplitudes $A(\tau)$ and for small false-alarm probabilities $F$.

\subsection{Periodicities of V352 CMa}

We discovered the following periodicities

$P_{\mathrm{w}}=7.24 \pm 0.22$ days $=$ Mean of rotation period

$P_{\mathrm{al}, 1}=7.158 \pm 0.002$ days $=$ Period of active longitudes

$P_{\mathrm{C}}=11.7 \pm 0.5 \mathrm{yr}=$ Activity cycle in $M(\tau)$ 
in the long-term photometry of V352 CMa. The scatter of the published age estimates is large (Table 1: between 33 and $6500 \mathrm{Myr}$ ). Combining $P_{\mathrm{w}}=7.24$ days to the colour index $B-V=0.705$ (Gaidos 1998; Cutispoto et al. 1999) gives a gyrochronological age of $295 \pm 36$ Myr for V352 CMa (Barnes 2007, Eqs. (3) and (16)). Gyrochronology can provide theoretical age estimates (e.g. Barnes 2010; Barnes \& Kim 2010), while the CoRot (do Nascimento et al. 2013) and the Kepler (do Nascimento et al. 2014) space missions provide the required accurate rotation measurements for solar analogs or "solar twins".

\section{Conclusions}

We wanted to test the performance of CPS by applying it to lowamplitude light curves of V352 CMa. This was challenging because the dispersions of the $\Delta V_{\mathrm{V}-\mathrm{C}}$ and $\Delta V_{\mathrm{K}-\mathrm{C}}$ magnitudes were comparable (Figs. 1a, b). From a total of 485 datasets, CPS failed to detect any periodicity in $178 \equiv 37 \%$ of the datasets.

Comparison of CPS and PSM revealed that both methods gave the same best periods for high amplitude to noise ratio light curves, but only if the correct model for the data was a sinusoid $(K=1)$. The periods detected were usually different for the $K=2$ models - even for the higher $A / N$ light curves. We conclude that incorrect periods $P(\tau)$ or model orders $K$ often lead to incorrect values for $M(\tau), A(\tau), t_{\min , 1}$ and $t_{\min , 2}$.

We detected signs of an activity cycle, $P_{\mathrm{C}}=11.7 \pm 0.5 \mathrm{yr}$ $(F=0.010)$, in the mean magnitudes $M(\tau)(n=39)$. However, this cycle is only $1.3 \mathrm{yr}$ shorter than the time span of the data, $\Delta T=13.0 \mathrm{yr}$.

The $P(\tau)$ period changes could be mostly spurious $\left(Z_{\mathrm{spu}}>\right.$ 0.15 ) due to low $A / N$ ratio (Lehtinen et al. 2011, Table 3). If the law of solar differential rotation were valid in V352 CMa, and these $P(\tau)$ changes traced this phenomenon, the surface differential rotation coefficient would be $|k|>Z_{\text {phys }} \approx 0.12$.

The Kuiper method detected an active longitude rotating with a period of $P_{\mathrm{al}, 1}=7.158 \pm 0.002(Q=0.004)$. This longlived structure was present between 1998 and 2009, but it vanished when the amplitudes of the light curves fell to near zero in 2010 .

Acknowledgements. This research at the Department of Physics (University of Helsinki) was performed in collaboration with the participants of the course "Variable stars", which was lectured in spring 2012. This work has made use of the SIMBAD database at CDS, Strasbourg, France and NASA's Astrophysics Data System (ADS) bibliographic services. The work by PK and JL was supported by the Vilho, Yrjö and Kalle Väisälä Foundation. The automated astronomy program at Tennessee State University has been supported by NASA, NSF, TSU and the State of Tennessee through its Centers of Excellence program.

\section{References}

Abt, H. A., \& Willmarth, D. 2006, ApJS, 162, 207

Andersen, J., Nordstrom, B., Ardeberg, A., et al. 1985, A\&AS, 59, 15
Árnadóttir, A. S., Feltzing, S., \& Lundström, I. 2010, A\&A, 521, A40 Baliunas, S. L., Donahue, R. A., Soon, W. H., et al. 1995, ApJ, 438, 269

Barnes, S. A. 2007, ApJ, 669, 1167

Barnes, S. A. 2010, ApJ, 722, 222

Barnes, S. A., \& Kim, Y.-C. 2010, ApJ, 721, 675

Beavers, W. I., \& Eitter, J. J. 1981, PASP, 93, 765

Beavers, W. I., \& Eitter, J. J. 1986, ApJS, 62, 147

Brugamyer, E., Dodson-Robinson, S. E., Cochran, W. D., \& Sneden, C. 2011, ApJ, 738, 97

Christian, D. J., Mathioudakis, M., Jevremovic, D., et al. 2003, Inform. Bull. Variable Stars, 5447, 1

Cutispoto, G., Pastori, L., Tagliaferri, G., Messina, S., \& Pallavicini, R. 1999, A\&AS, 138, 87

Decin, G., Dominik, C., Malfait, K., Mayor, M., \& Waelkens, C. 2000, A\&A, 357,533

do Nascimento, Jr., J.-D., Takeda, Y., Meléndez, J., et al. 2013, ApJ, 771, L31

do Nascimento, Jr., J.-D., García, R. A., Mathur, S., et al. 2014, ApJ, 790, L23

Eggen, O. J. 1995, AJ, 109, 1327

Fekel, F. C., \& Henry, G. W. 2005, AJ, 129, 1669

Fernandes, J. M., Vaz, A. I. F., \& Vicente, L. N. 2011, A\&A, 532, A20

Gaidos, E. J. 1998, PASP, 110, 1259

Gaidos, E. J., \& Gonzalez, G. 2002, New Astron., 7, 211

Gaidos, E. J., Henry, G. W., \& Henry, S. M. 2000, AJ, 120, 1006

Gontcharov, G. A. 2006, Astron. Lett., 32, 759

Gray, R. O., Corbally, C. J., Garrison, R. F., et al. 2006, AJ, 132, 161

Hackman, T., Mantere, M. J., Jetsu, L., et al. 2011, Astron. Nachr., 332, 859

Hackman, T., Pelt, J., Mantere, M. J., et al. 2013, A\&A, 553, A40

Haywood, M. 2001, MNRAS, 325, 1365

Henry, G. W. 1999, PASP, 111, 845

Holmberg, J., Nordström, B., \& Andersen, J. 2009, A\&A, 501, 941

Horne, J. H., \& Baliunas, S. L. 1986, ApJ, 302, 757

Jeffries, R. D., \& Jewell, S. J. 1993, MNRAS, 264, 106

Jetsu, L., \& Pelt, J. 1996, A\&AS, 118, 587

Jetsu, L., Hackman, T., Hall, D. S., et al. 2000, A\&A, 362, 223

Kajatkari, P., Hackman, T., Jetsu, L., Lehtinen, J., \& Henry, G. W. 2014, A\&A, 562, A107

Koen, C., \& Eyer, L. 2002, MNRAS, 331, 45

Kóspál, Á., Ardila, D. R., Moór, A., \& Ábrahám, P. 2009, ApJ, 700, L73

Lehtinen, J., Jetsu, L., Hackman, T., Kajatkari, P., \& Henry, G. W. 2011, A\&A, 527, A136

Lehtinen, J., Jetsu, L., Hackman, T., Kajatkari, P., \& Henry, G. W. 2012, A\&A, 542, A38

Maldonado, J., Martínez-Arnáiz, R. M., Eiroa, C., Montes, D., \& Montesinos, B. 2010, A\&A, 521, A12

Martínez-Arnáiz, R., Maldonado, J., Montes, D., Eiroa, C., \& Montesinos, B. 2010, A\&A, 520, A79

Nakajima, T., \& Morino, J.-I. 2012, AJ, 143, 2

Nakajima, T., Morino, J.-I., \& Fukagawa, M. 2010, AJ, 140, 713

Nordström, B., Mayor, M., Andersen, J., et al. 2004, A\&A, 418, 989

Raghavan, D., McAlister, H. A., Henry, T. J., et al. 2010, ApJS, 190, 1

Rodonò, M., Messina, S., Lanza, A. F., Cutispoto, G., \& Teriaca, L. 2000, A\&A, 358,624

Santos, N. C., Israelian, G., \& Mayor, M. 2001, A\&A, 373, 1019

Santos, N. C., Israelian, G., García López, R. J., et al. 2004a, A\&A, 427, 1085

Santos, N. C., Israelian, G., Randich, S., García López, R. J., \& Rebolo, R. 2004b, A\&A, 425, 1013

Shara, M. M., Shara, D. J., \& McLean, B. 1993, PASP, 105, 387

Wright, J. T., Marcy, G. W., Butler, R. P., \& Vogt, S. S. 2004, ApJS, 152, 261 